\title{
Deficiency of maize starch-branching enzyme $i$ results in altered starch fine structure, decreased digestibility and reduced coleoptile growth during germination
}

\author{
Huan Xia ${ }^{1,3}$, Marna Yandeau-Nelson ${ }^{2,4}$, Donald B Thompson ${ }^{3}$ and Mark J Guiltinan ${ }^{4 *}$
}

\begin{abstract}
Background: Two distinct starch branching enzyme (SBE) isoforms predate the divergence of monocots and dicots and have been conserved in plants since then. This strongly suggests that both SBEI and SBEIl provide unique selective advantages to plants. However, no phenotype for the SBEI mutation, sbela, had been previously observed. To explore this incongruity the objective of the present work was to characterize functional and molecular phenotypes of both sbela and wild-type (Wt) in the W64A maize inbred line.

Results: Endosperm starch granules from the sbela mutant were more resistant to digestion by pancreatic $\alpha$ amylase, and the sbela mutant starch had an altered branching pattern for amylopectin and amylose. When kernels were germinated, the sbela mutant was associated with shorter coleoptile length and higher residual starch content, suggesting that less efficient starch utilization may have impaired growth during germination.

Conclusions: The present report documents for the first time a molecular phenotype due to the absence of SBEl, and suggests strongly that it is associated with altered physiological function of the starch in vivo. We believe that these results provide a plausible rationale for the conservation of SBEl in plants in both monocots and dicots, as greater seedling vigor would provide an important survival advantage when resources are limited.
\end{abstract}

\section{Background}

The starch granule is a highly-ordered structure with alternating crystalline and amorphous growth rings $[1,2]$. Starch molecules are biopolymers of anhydroglucose units linked by $\alpha-1,4$ and $\alpha-1,6$ glycosidic bonds. They are composed of two glucan polymers, the generally linear fraction, amylose, and the branched fraction, amylopectin. The constituent amylopectin chains can be mainly categorized into A chains (not bearing any branches) and B chains (bearing one or more branches) [3]. The main physiological functions of starch include high-density storage of energy and the controlled release of this energy during starch degradation.

Starch-branching enzyme (SBE) plays an important role in starch biosynthesis by introducing branch points,

\footnotetext{
* Correspondence: mjg9@psu.edu

${ }^{4}$ Department of Horticulture, The Pennsylvania State University, University

Park, Pennsylvania 16802-5807, USA

Full list of author information is available at the end of the article
}

the $\alpha-1,6$ linkages in starch. Boyer and Preiss [4] identified three major SBE isoforms in developing maize kernels: SBEI, SBEIIa, and SBEIIb. The SBE isoforms have been shown to be encoded by different genes [5-8]. Phylogenetic analyses of SBE sequences from a number of plant species have shown that the SBEI and SBEII isoforms are conserved among most plants, and that SBEIIa and SBEIIb isoforms are conserved among most monocots [9-13]. Furthermore, genes belonging to both the SBEI and SBEII families can be identified in various lineages of green alga, which supports the theory that these two families of genes evolved approximately a billion years ago [14]. These examples of extreme evolutionary conservation are strong evidence for a specific and vital role for each enzyme isoform in starch biosynthesis.

In vitro biochemical analyses have documented that the SBEI and SBEII isoform activities are not identical $[15,16]$, but these studies do not necessarily indicate

\section{Ciomed Central}


their action in vivo, as starch biosynthesis occurs in the presence of starch synthases and debranching enzymes. Studies have suggested that multi-protein starch synthesizing complexes exist, and that interactions within these complexes could modulate the intricate structure of a developing starch granule [17-33]. Whether there are functional differences among SBE isoforms in vivo remains to be addressed.

Insight into a possible in vivo function of an SBE may be gained from the study of sbe mutants deficient in one or more SBE isoform activities. The maize amylose extender (ae) mutant, which is deficient in SBEIIb, has a profound effect on starch structure, leading to an increased amylose proportion and a reduced branching density of endosperm amylopectin [5,33-35]. More recently, studies of a maize sbe $2 a$ mutant showed that deficiency of the SBEIIa isoform decreased plant fitness and resulted in lower kernel yield, but there was minimal effect on kernel starch properties $[11,36]$. Previous work showed no effect of SBEI deficiency (in the sbe1a mutation) on starch molecular size and on chain length distribution after debranching [26,37]. Subsequently, preliminary analysis of susceptibility of sbe1a endosperm starch to pancreatic $\alpha$-amylase digestion, using the AOAC procedure (2002.02) to determine enzyme-resistant starch (RS), indicated that sbe1a mutant endosperm starch had a greater resistance to digestion [38]. We reasoned that it was likely that the deficiency in SBEI led to reduced susceptibility to enzymatic digestion by altering the starch structure in some way. Thus, in this work we sought to confirm this initial observation and to explore more subtle aspects of starch structure in the sbe1a mutant. The objective of the present work was to characterize functional and molecular phenotypes of both sbe1a and wild-type (Wt) in the W64A maize inbred line.

\section{Results}

\section{Starch Molecular Structure}

To study the functional role of SBEI on molecular structure of amylose and amylopectin, Wt Sbe1a starch and mutant sbe1a starch were fractionated from mature kernels. The maize sbe1a mutant contains a $M u$ transposon in the 14th exon of the Sbe1a gene, and was previously shown to be null for the expression of SBEI transcript and protein [37]. The proportions, iodine binding properties, and size-exclusion chromatograms for the amylopectin and amylose fractions were similar for Wt and sbe1a starch (data not shown). To study the molecular fine structure, $\beta$-amylolysis and subsequent isoamylase and pullulanase debranching were applied to both the amylopectin and amylose fractions from Wt and sbela. Despite a similar chain length (CL) profile observed for both fractions from the two genotypes (see Additional
File 1 online), the CL distribution after various extents of $\beta$-amylolysis showed differences for Wt and sbe1a (Figure 1A; see Additional File 2 online).

For the amylopectin fraction from both genotypes, hydrolysis with $\beta$-amylase caused a dramatic change in CL distribution within the first $10 \mathrm{~min}$ (Figure 1A): A major increase was observed below degree of polymerization $(\mathrm{DP}) \sim 10$. In this region for $\mathrm{Wt}$, the change in the CL distribution from $10 \mathrm{~min}$ to $24 \mathrm{~h}$ of $\beta$-amylolysis was primarily a reduction of the DP 4 stubs to DP 2 stubs; however, for the sbela sample no further reduction in DP 4 was observed after 10 min (Figure 1A). After $24 \mathrm{~h}$ of $\beta$-amylolysis, conditions necessary to produce $\beta$-limit dextrin ( $\beta$-LD) $[39,40]$, the sbe1a sample had a much smaller proportion of the DP 2 chains and a much larger proportion of DP 4 chains than the Wt sample (Figure 1A; see Additional File $2 \& 3$ online).

For the amylose fraction from both genotypes, $\beta$-LD was produced. Analysis of the CL distribution of isoamylase-debranched $\beta$-LDs showed a higher proportion of chains of DP $\geq 100$ and lower proportions of other chains (DP < 100), before and after pullulanase addition (Figure 1B; see Additional File 4 online). The subsequent pullulanase debranching led to an increase in both the DP 3 and DP 2 areas for both genotypes, and this increase was greater in sbe1a (Figure 1B; see Additional File 4 online). The subsequent pullulanase debranching also led to a decrease in chains of approximately DP 8-9 for both genotypes (Figure 1B).

\section{Starch Digestibility In vitro by Pancreatic $\alpha$-Amylase}

Starch hydrolysis is an important feature of starch function both in the plant and when the plant is used for human food. Hydrolysis of starch ingested as food can vary both with respect to the rate and the extent of digestion by pancreatic $\alpha$-amylase. In the human digestive tract, the undigested starch that reaches the colon is termed RS; the level of RS is a measure of the extent of digestion by this enzyme. An official in vitro method (AOAC 2002.02) is used for determination of the RS level. This method was modified to allow study of both the digestion rate and the extent of digestion [41,42]. Ftests performed for a fully nested analysis of variance (ANOVA) showed an effect of genotype $(p=0.000)$, but no effect of biological replication $(p=0.334)$. The RS value was higher in the sbe1a mutant starch (13.2\%) than in the Wt starch (1.6\%) from measures of 3 biological replications $(p<0.05)$.

The digestion pattern was similar among the three biological replications for each genotype (data not shown). For graphic illustration of the digestion timecourse, curves for one biological replication for each genotype are shown in Figure 2. The kinetics of digestion were analyzed using a five-parameter, double- 

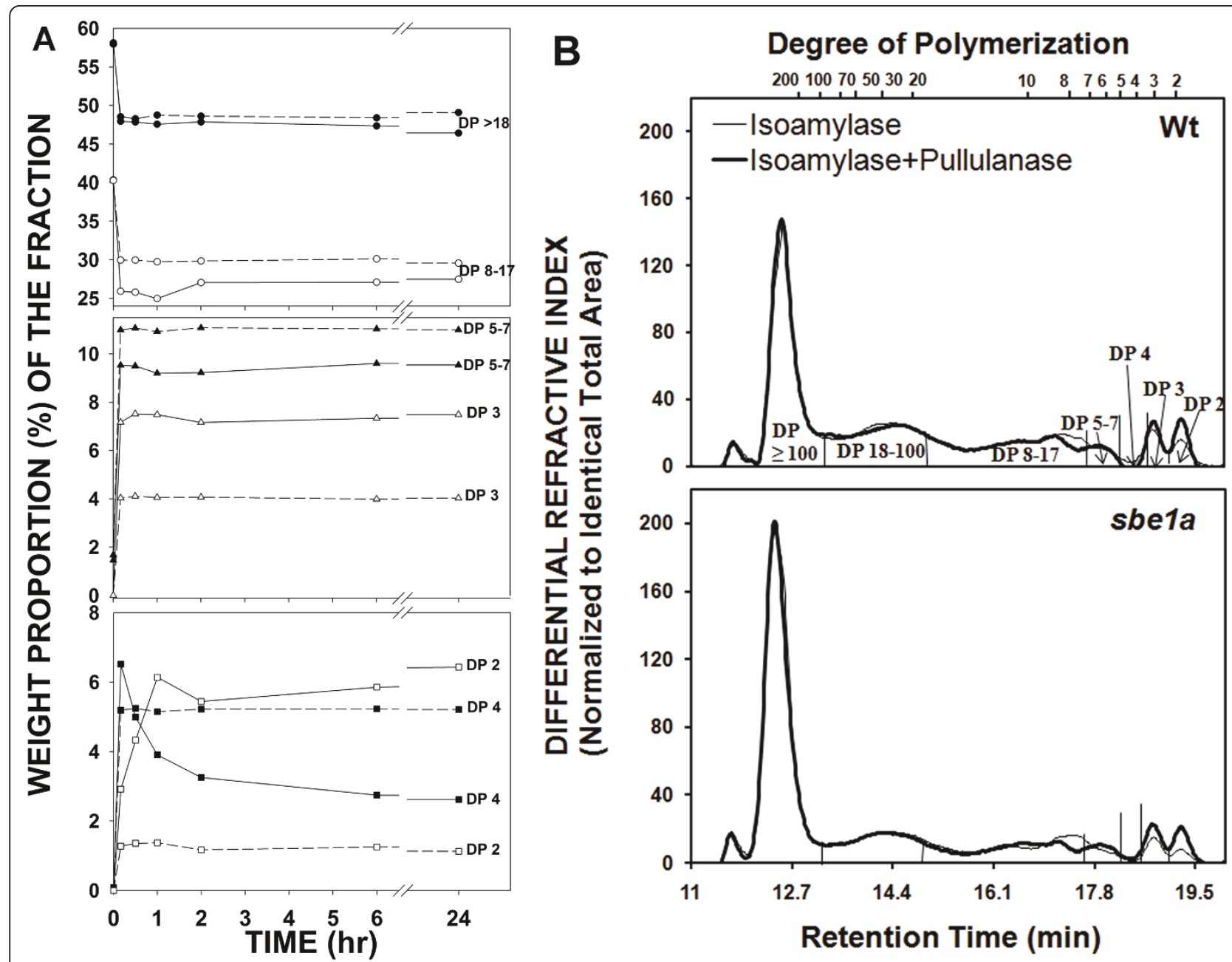

Figure 1 Amylopectin and amylose structure of Wt and sbe1a mutant starch samples by HPSEC analysis. A. Proportions of chains ${ }^{1}$ from debranched ${ }^{2} \beta$-dextrins during time course of $\beta$-amylolysis of amylopectin from Wt ( - -) and sbela mutant (- - ) starch using $\beta$-amylase (250 $\mathrm{U} / \mathrm{mL}$ ). B. Chromatograms ${ }^{1}$ of isoamylase-debranched and isoamylase-plus-pullulanase-debranched $\beta$-limit dextrins ${ }^{3}$ from amylose fraction from Wt and sbela mutant starch. 'Chromatographic regions were divided as in [40]. Proportions of DP $\geq 18, D P$ 8-17, DP 5-7, DP 4, DP 3 and DP 2 were calculated as the areas for $D P \geq 17.5,7.5 \leq D P \leq 17.5,4.5 \leq D P \leq 7.5,3.5 \leq D P \leq 4.5,2.5 \leq D P \leq 3.5$, and $D P \leq 2.5$, respectively, as in [40].

Proportions of chains in each region for $\mathbf{B}$ are presented in Additional File 4. Calculation was based on representative chromatograms for starch from one biological replication. Values are percentage by weight. ${ }^{2}$ Debranching was performed successively with isoamylase for $24 \mathrm{~h}$ and pullulanase for 24 h. ${ }^{3} \beta$-Limit dextrin was obtained after 3 times of $24-h \beta$-amylolysis on amylose.

exponential decay model (see "Materials and Methods"), and the calculated parameters are presented in Table 1. A higher $y_{0}$ (the limit of digestion as determined using the model) was found in sbe1a than for Wt (Table 1), consistent with the higher limit of digestion given by the RS value for this genotype.

\section{Granular Morphology of Native Starch and Residual Starch after Digestion}

Scanning electron microscopy was used to image starch granules from Wt and sbela mutant plants in native form and after the $16 \mathrm{~h}$ in vitro digestion with pancreatic $\alpha$-amylase for determination of the RS value. Prior to digestion, native starch granules from Wt and sbe1a had similar morphology (Figure 3A) with an average diameter of $10.2 \mu \mathrm{m}$ for Wt and $9.8 \mu \mathrm{m}$ for sbe1a. However, after digestion, differences were observed between the two genotypes (Figure 3B; see Additional File 5 \&6 online). Samples of the sbela RS contained many residual granules with distinct holes in the surface and hollow interiors, whereas for Wt only small fragments of residual granules were seen (Figure 3B). The Wt fragments also showed evident alternating layers on the edge of the pieces, which was less evidently present in sbe1a samples (Figure 3B; see Additional File 6 online).

Light micrographs of iodine-stained native granules are shown in Figure 3C. For both genotypes, all native starch granules were stained blue and produced a 


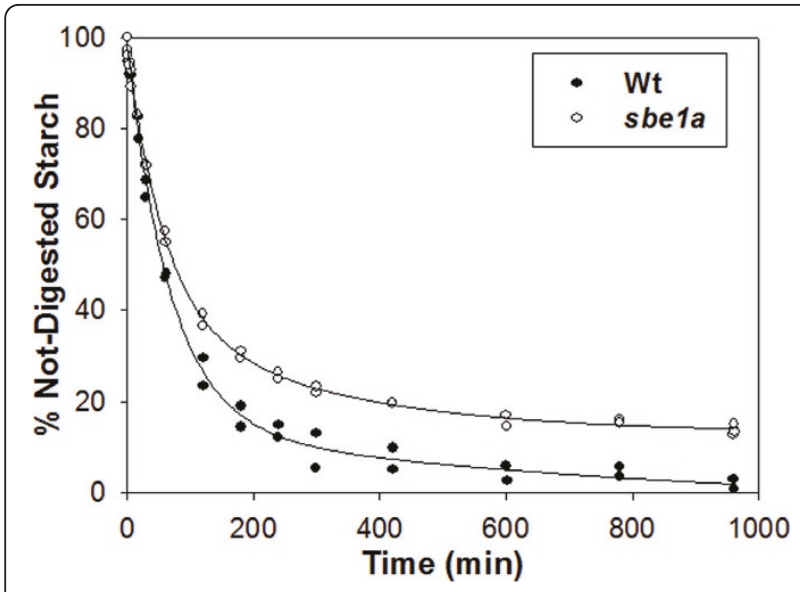

Figure 2 Time-course of digestion of the resistant starch assay for Wt and sbe1a mutant starch. Results shown were from one biological replication. Curves shown are best fits of analysis of combined data from two independent digestions.

characteristic Maltese Cross when viewed in the polarized light microscope; however, sbe1a native starch showed more heterogeneity in staining as compared to $\mathrm{Wt}$, as there were more relatively dark-stained granules in sbe1a than in Wt native starch (24.3\% and 8.7\%).

\section{Starch Utilization during Kernel Germination}

As endosperm starch from the sbe1a mutant has a lower susceptibility to pancreatic $\alpha$-amylase, we suspected that the sbe1a endosperm starch might be less readily utilized during kernel germination. To study the effect of sbe1a on kernel germination, starch utilization and coleoptile growth during germination of Wt and sbe1a mutant kernels were examined.

All the kernels from three different ears of both Wt and sbe1a genotypes were germinated, demonstrating no

Table 1 Kinetics of digestion ${ }^{1}$ of the resistant starch assay for Wt and sbe1a mutant starch ${ }^{2}$

\begin{tabular}{lccccc}
\hline Starch & $\boldsymbol{y}_{\mathbf{o}}(\%)$ & $\boldsymbol{S}_{\mathbf{1}}(\%)$ & $\boldsymbol{k}_{\mathbf{1}}\left(\mathbf{m i n}^{-\mathbf{1}}\right)$ & $\boldsymbol{S}_{\mathbf{2}}(\%)$ & $\boldsymbol{k}_{\mathbf{2}}\left(\mathbf{m i n}^{-\mathbf{1}}\right)$ \\
\hline $\mathbf{W t}$ & $-5.4 \pm$ & $85.9 \pm$ & $1.4 \pm 0.1^{\mathrm{a}}$ & $17.9 \pm$ & $0.9 \pm 0.2^{\mathrm{a}}$ \\
& $2.3^{\mathrm{a}}$ & $3.5^{\mathrm{b}}$ & $\left(\times 10^{-2}\right)$ & $5.4^{\mathrm{a}}$ & $\left(\times 10^{-3}\right)$ \\
sbe1a & $13.7 \pm$ & $59.8 \pm$ & $1.8 \pm 0.1^{\mathrm{b}}$ & $24.3 \pm$ & $3.0 \pm 1.1^{\mathrm{b}} \pm$ \\
& $2.8^{\mathrm{b}}$ & $3.0^{\mathrm{a}}$ & $\left(\times 10^{-2}\right)$ & $2.4^{\mathrm{a}}$ & $\left(\times 10^{-3}\right)$ \\
\hline
\end{tabular}

\footnotetext{
${ }^{1}$ Kinetic parameters are obtained from model fit using the double exponential decay equation:

$$
y=y_{0}+S_{1} e^{-k_{1} x}+S_{2} e^{-k_{2} x}
$$

where $y$ is $\%$ NDS, $x$ is the time, $y_{0}$ is the $y$-value that the model asymptotically approaches, $S_{1}$ and $S_{2}$ are the concentrations of the two different substrate components, and $k_{1}$ and $k_{2}$ are the reaction rate constants for the decay of the two different components.

${ }^{2}$ Values are expressed as mean \pm SD for three biological replications. Values for each biological replication were obtained from fit of combined data from two independent digestions. Significant differences $(p<0.05)$ in the same column, as determined by one-way ANOVA analysis, are indicated by different superscripts.
}

differences in germination rate. The coleoptile length of each genotype was measured daily over 11 days (Figure 4). The average length of sbe1a coleoptiles was shorter than Wt from Day 7 onward (Figure 4). For both genotypes the endosperm starch content decreased over time (Figure 4). On Days 6, 8, and 11, the starch content was higher in sbe1a germinating endosperm as compared to Wt, suggesting less utilization of starch. This trend is consistent with the reduced growth of sbe1a coleoptiles after Day 6 .

\section{Discussion}

\section{Starch Molecular Structure}

In the present study, rapid degradation of chains DP $\geq 18$ and DP 8-17 were observed for both Wt and sbe1a samples in the first $10 \mathrm{~min}$ of $\beta$-amylolysis (Figure 1A). As $\beta$-amylase cannot bypass branch points to hydrolyze starch chains, a plausible interpretation for the less extensive degradation of DP 8-17 in sbe1a would be that the B chains (those chains with other chains attached) [43] would have slightly longer internal segments and shorter external chains. For the second stage of $\beta$-amylolysis [44], a slow reduction in the amount of DP 4 chains was observed in Wt samples over the period of $10 \mathrm{~min}$ to $24 \mathrm{~h}$ but not in sbela samples (Figure $1 \mathrm{~A})$, suggesting differences in the proportion of branch points that would differentially limit access of the enzyme to glycosidic linkages [40].

Amylopectin branching pattern models for both sbe1a and Wt are presented to account for this difference in $\beta$-amylase action on DP 4 stubs (Figure $5 \mathrm{~A}$ ). In the model for sbe1a, DP 4 stubs would be difficult for $\beta$ amylase to hydrolyze to DP 2 when closely associated branch points present a steric barrier to binding of $\beta$ amylase. Although most of the DP 4 is from residual A chains [43], some DP 4 chains from residual B chains would result from short $B$ chains with short internal segments. The incomplete hydrolysis of DP 4 in sbe1a suggests that A chains are preferentially localized near another branch point, leading to 1) hindered hydrolysis of residual A chains of DP 4 to DP 2 due to steric constraint, and 2) more residual B chains with DP 4 due to incidence of short internal segments (Figure 5A). In the model for Wt, the DP 4 stubs would be slowly hydrolyzed to DP 2, as there is less steric hindrance from proximal branch points. According to the two models, sbe1a amylopectin contains a higher proportion of closely associated branch points than Wt. Furthermore, based on CL profiles (see Additional File 1 online), the calculated overall average branching density is similar in the two amylopectins. Thus, we suggest that the effect of the sbela mutation is to increase the local concentration of branch points but not to influence the overall amount of branch points in amylopectin. 

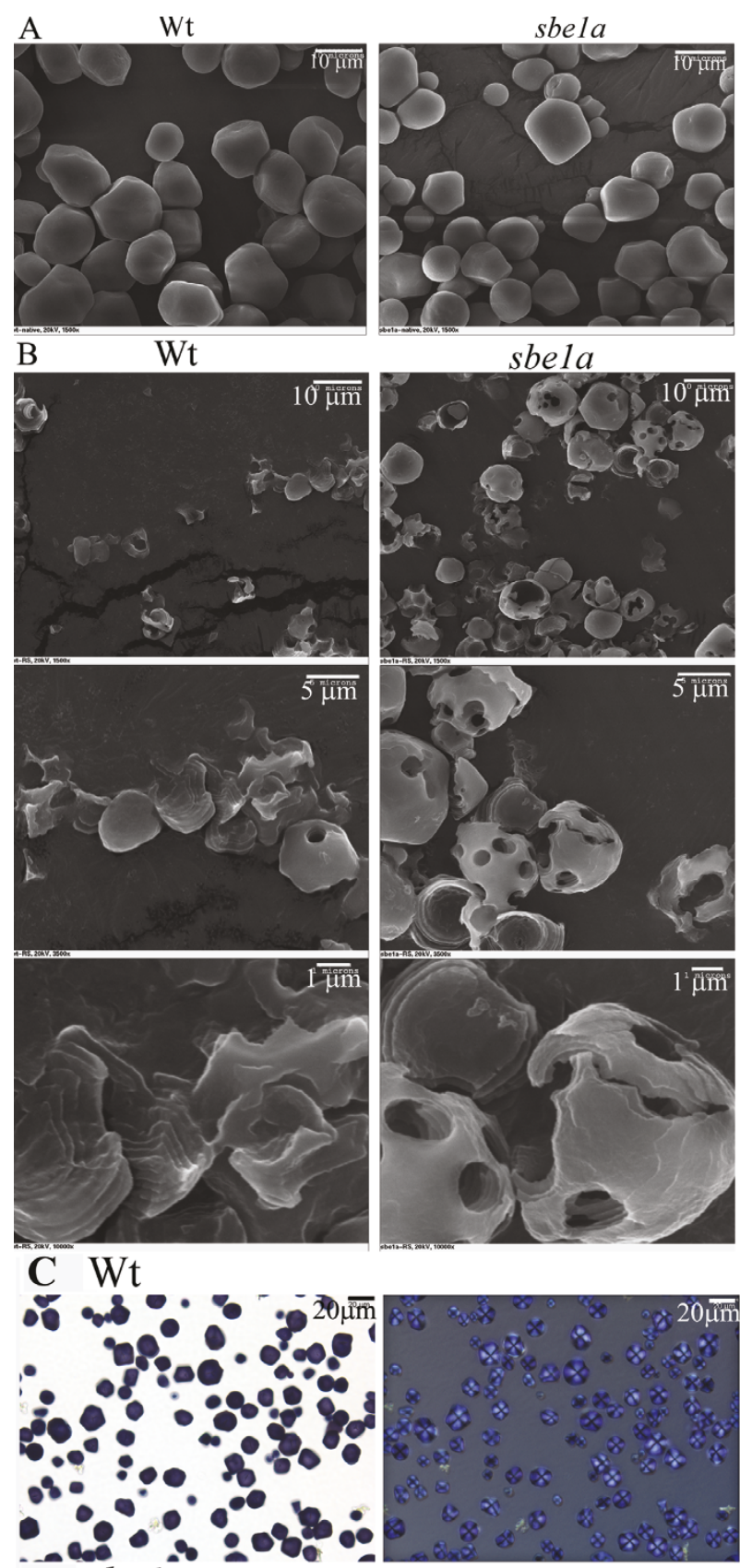

sbela

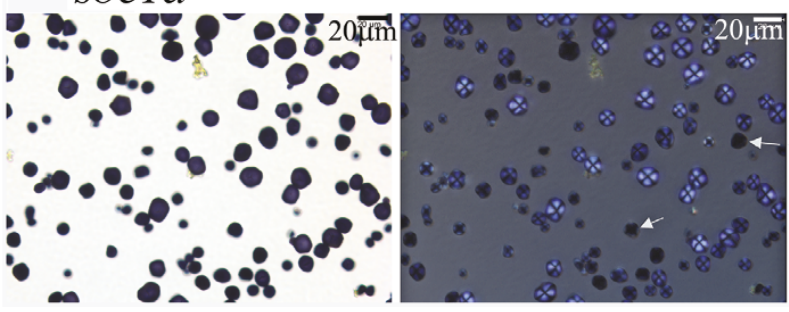

Figure 3 Micrographs of Wt and sbe1a mutant starch samples. A. Scanning electron micrographs of native starch from Wt (left) and sbela mutant (right). Scale bars represent $10 \mu \mathrm{m}$ at the top of the graphs. B. Scanning electron micrographs of residual starch after 16-h $\alpha$-amylase digestion from Wt and sbela mutant. Scale bars represent $10 \mu \mathrm{m}, 5 \mu \mathrm{m}$, or $1 \mu \mathrm{m}$ at the top of the graphs. C. Bright field (left) and polarized light (right) micrographs of native Wt and sbela mutant starch. The specimen were stained with $0.04 \%$ iodine and viewed within 5 min. Arrows point to dark stained granules. 


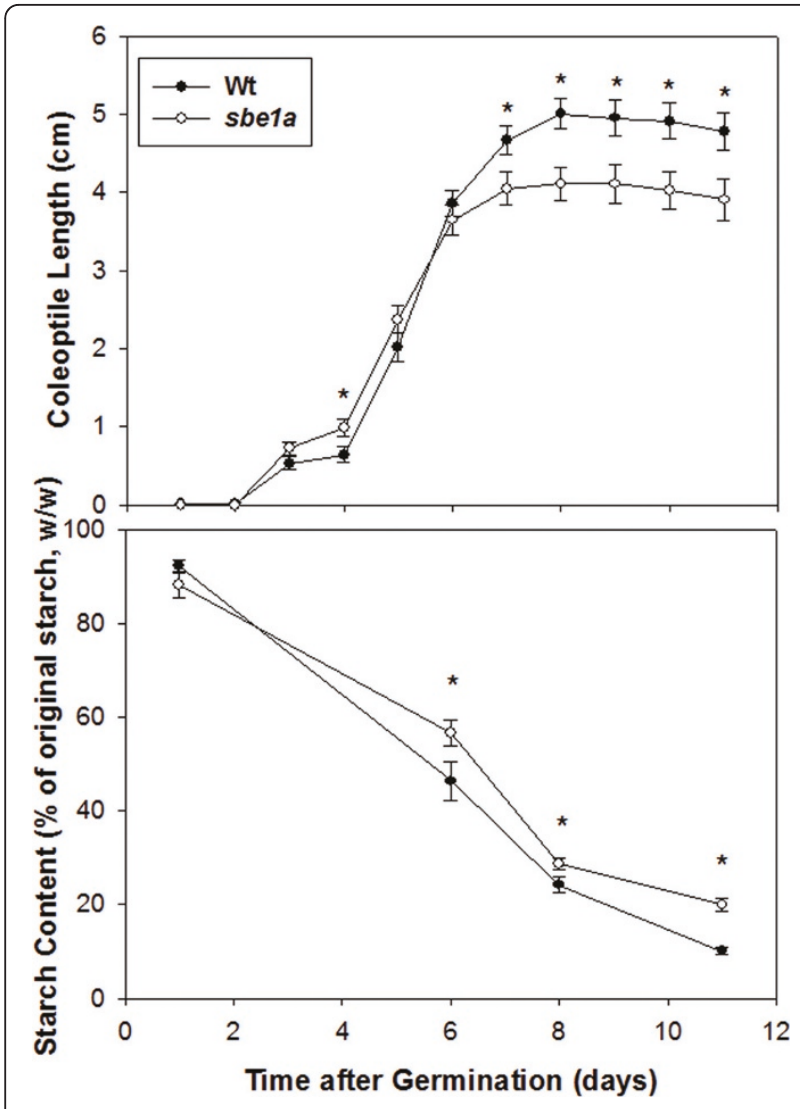

Figure 4 Germination analysis of $\mathrm{Wt}$ and sbe1a mutant kernels The lengths of the emerged coleoptiles were measured on successive days during the incubation period ${ }^{1}$. Starch content in the germinating endosperm was quantified at Day $1,6,8,11$, and percentage of starch content at each day against the dry weight of Day 1 kernels was plotted'. 'Each data point is mean \pm standard error of measurements of kernels from three biological replications. As 2 kernels were removed at Day 1, 6, 8, 11 for quantifying starch content, 15, 13, 11, and 9 kernels from three biological replications were used for coleoptile measurement Day 1, 2-6, 7-8, 9-11, respectively. Comparison between two genotypes for each day was made by one-way ANOVA analysis and a significant difference was marked by an asterisk $(p<0.05)$.

In the debranched $\beta$-LDs from the amylose fraction (but not in intact amylose), a higher proportion of long chains of DP $\geq 100$ was observed in sbe1a (Figure 1B and Additional File 1 online). The higher proportion of longer chains in $\beta$-LDs of amylose from sbe1a can be explained by branch points that tend to be closer to the non-reducing ends, so that longer internal chains result.

When debranching of $\beta$-LDs from amylose was performed with isoamylase without subsequent pullulanase digestion, there were fewer DP 2 than DP 3 chains (Figure 1B; see Additional File 4 online). For $\beta$-LD from amylopectin, all of the DP 3 and some of the DP 2 chains are known to be debranched by isoamylase [40]. However, our results of $\beta$-LD from amylose for both

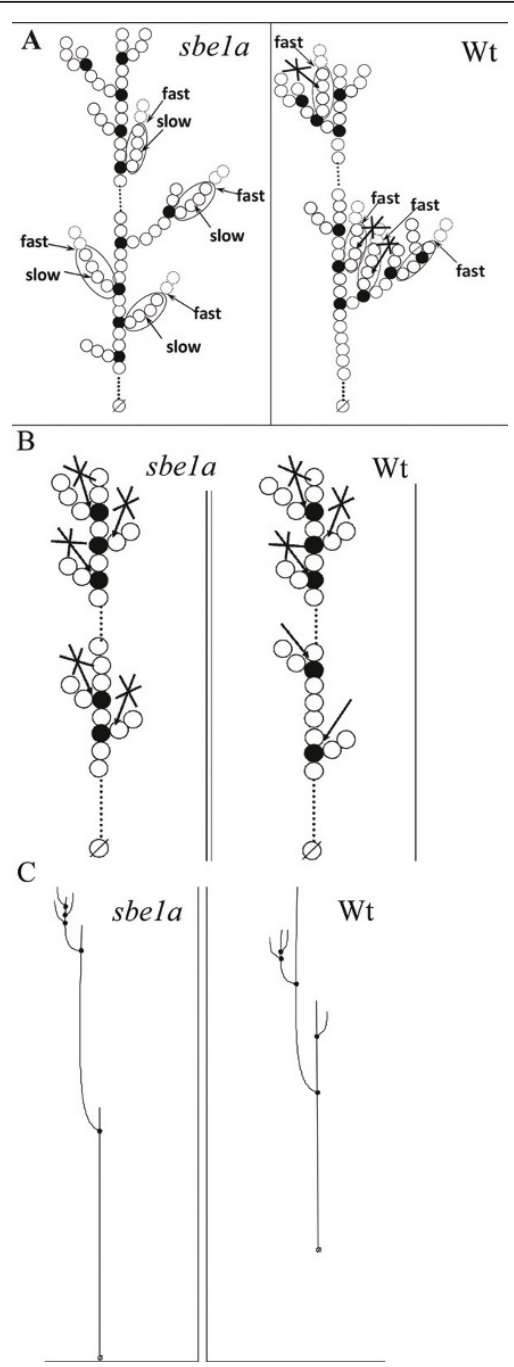

Figure 5 Branching pattern models. A. Branching pattern models for amylopectin from sbe1a and Wt starches. Shown are b-dextrins approaching the limit of digestion by $\beta$-amylase, with differences in the amount of DP 4 stubs. All circles indicate glucose units. Dotted line indicates more glucose units. Dotted circles indicate glucose hydrolyzed by $\beta$-amylase. Solid black circles indicate branch points. Circles with a slash indicate reducing ends. Circles in an ellipse indicate glucose units that would result in a DP 4 chain. Arrows indicate the action sites of $\beta$-amylase. Arrows with a cross indicates that action of $\beta$-amylase is prevented by closely associated branch points nearby. Fast and slow indicate the first and second stage of $\beta$-amylolysis, respectively. B. Branching pattern models for a region of the amylose from sbe1a and Wt starches. Shown are $\beta$-limit dextrins that are consistent with difference in action of isoamylase. All circles indicate glucose units. Dotted lines indicate more glucose units. Solid black circles indicate branch points. Circles with a slash indicate reducing ends. Arrows indicate the action sites of isoamylase. Arrows with a cross indicates that action of isoamylase is prevented by closely associated branch points nearby. The model does not consider the presence of B chains. C. Proposed overall amylose branching pattern models for sbe1a and Wt starches, consistent with the differences in actions of $\beta$-amylase and isoamylase. All lines indicate glucose chains. Solid black circles indicate branch points. Circles with a slash indicate reducing ends. 
genotypes suggest that even some DP 3 chains are not debranched by isoamylase. Comparing sbe1a to Wt, more of DP 2 and DP 3 chains are not debranched by isoamylase in $\beta$-LD from sbe1a amylose (Figure 1B). As the structures escaping isoamylase debranching may have closely associated branch points and those structures can be debranched by pullulanase [40], a greater increase in both DP 2 and DP 3 by subsequent pullulanase treatment suggests that a higher proportion of these structures are resistant to isoamylase in amylose from sbe1a. Amylose branching pattern models are presented in Figure 5B to account for the difference in isoamylase action. In the model for sbe1a, A chains are preferentially attached by branch points close to each other whereas in Wt, A chains are not, leading to less hindered isoamylase debranching.

Our data suggest that amylose of sbe1a mutant starch has 1) longer internal chains and 2) more A chains attached by branch points close to each other. This evidence can be used to create an overall model for amylose branching patterns of sbe1a and Wt (Figure 5C). The models are drawn taking into account similar CL profiles (see Additional File 1 online) and assuming that $\sim 50 \%$ of amylose molecules are branched, with $\sim 5-6$ branches per molecule [45]. According to the proposed model, for sbe1a, A chains are closer to each other, and the location of the chains tends to be more towards non-reducing end. For Wt, A chains are farther away from each other, and the location of the chains is more random and thus more distributed.

\section{Starch Digestion}

Kinetic analysis shows that the $y_{0}$ value for Wt starch is effectively zero (Table 1 ), in agreement with the RS value for Wt starch (1.6\%), and the $y_{0}$ and RS values for sbe1a starch are also in good agreement.

The kinetic model is based on the presence of two general types of starch substrate: a rapidly-digested substrate $\left(S_{1}\right)$, and a slowly-digested substrate $\left(S_{2}\right)[41,42]$. The two genotypes differ both in the proportions of $S_{1}$ and $S_{2}$ and the reaction rate constants for these two components. The $S_{1}$ components of Wt and sbe1a starch were $85.9 \%$ and $59.8 \%$ respectively. This suggests that the sbe1a mutation altered the starch structure and this resulted in less rapidly-digested component. Consistent with our results, Ao et al. [46] found that increased branch density led to a decreased proportion of RDS (analogous to our $S_{1}$ ) and an increased proportion of SDS (analogous to our $S_{2}$ ).

\section{Starch Granular Structure}

Two microscopic techniques, scanning electron microscopy (SEM) and light microscopy (LM), were employed to observe granular structure before and after RS digestion by pancreatic $\alpha$-amylase. Native starch granules from Wt and sbe1a appear similar in size, shape, degree of birefringence, and morphology, as described in a previous report for $w x$ and sbe1a $w x$ granules [47]. Polarized light microscopy (see Additional File 5 online) shows that almost all of the digested Wt granules had lost their birefringence, while for sbe1a, many digested granules had maintained some birefringence in the peripheral area of the granules, which indicates that the center of the digested sbela granules is either gone or no longer crystalline enough to show birefringence. The presence of a hollow interior in the digested sbela granules was confirmed by SEM (Figure 3B), indicating a relatively greater resistance to digestion for the exterior portion of the sbe1a granule.

Most of the recovered RS from Wt were represented by small granule fragments. However, the sbe1a RS showed variations in morphology, from small fragments to hollow granules. The difference in digestion of individual granules may be due to differences in heterogeneity in granule structure, as a higher proportion of relatively dark-stained granules were observed in sbe1a than in Wt native starch (Figure $3 C$ ). SEM revealed the presence of alternating layers in the Wt residual fragments (Figure 3B), which probably reflect the residual growth rings after digestion.

By observing the sbe1a RS by SEM (Figure 3B), one may roughly estimate that, for the recovered granules, approximately $40 \%$ of granule content has escaped digestion. However the RS value for sbe1a starch is approximately $13 \%$. Therefore, some of the sbe1a granules were likely to have been digested completely. The heterogeneity found among sbe1a granules (Figure 3C) may account for different degree of digestion of individual granules. Thus, it can be reasoned that the micrographs of the sbe1a RS may disproportionately represent the more resistant granules.

A distinct feature of the recovered sbela RS is the presence of holes on the surface of the peripheral portion of the granules. These holes are possibly from the enlargement of the surface pores in native granules by $\alpha$-amylase hydrolysis [48]. The presence of these holes on the shell is consistent with previous studies demonstrating that digestion of normal granules starts with surface pores and proceeds through deeper hydrolysis in channels [49-52], followed by fragmentation [48]. In the current study, the presence of remaining shells with holes in the sbe1a RS indicates continuing difficulty in digestion by $\alpha$-amylase. Neither holes nor shells were observed in the Wt RS, indicating a more complete digestion.

As observed under microscopy, the RS from Wt consists mostly of portions of residual growth rings, while the RS of sbe1a is mostly residual peripheral regions. 
The kinetic analysis shows that the digestion of sbe1a starch reached a plateau by $16 \mathrm{~h}$, suggesting the RS from sbe1a is not further digested. When the RS is observed by SEM, one can conclude that some of the peripheral regions in sbela starch granules cannot be further digested. Enrichment of amylose has been found by some to exist toward the granule peripheral region $[53,54]$. SEM showed that the peripheral regions were more resistant to $\alpha$-amylase digestion in sbe1a granules. It is possible that these differences may be preferentially localized in the peripheral region of the granules, where starch synthesis may be more influenced by deficiency of SBEI [10]. The CL distribution of residual starch collected after $\alpha$-amylase digestion showed some small differences between Wt and sbe1a (see Additional File 7 online). However, no direct evidence was obtained in the current study about whether the molecular structure in the peripheral regions was different in sbe1a.

\section{Starch Utilization during Kernel Germination}

An endogenous $\alpha$-amylase is considered to be responsible for attacking the starch granule and initiating starch hydrolysis in germinating cereal endosperm [55]. Starch hydrolysis continues by the action of limit dextrinase, $\alpha$ amylase, $\beta$-amylase, and $\alpha$-glucosidase to produce maltose and glucose for plant utilization [55]. The observed reduction in starch hydrolysis during the later stages of germination raises the possibility that continued hydrolysis of $\alpha$-amylase-hydrolyzed glucans is hindered in the sbe1a mutant. The altered carbon metabolism could then cause a deficiency in general plant growth characteristics such as coleoptile length [23]. The structural analysis of sbe1a starch suggests that the decreased starch utilization of sbe1a seeds is due to an altered starch branching pattern.

\section{Consideration of SBEI Function in the Context of Pleiotropic Effects}

Differences in SBE activity in sbe mutants could be simply due to the amount of a remaining SBE isoform or to biochemical or physical interactions that modulate the activities of an isoform; for the latter possibility SBEI may be regulated through complex interactions with other starch synthetic enzymes. Colleoni et al [21] showed that two migratory forms of SBEI are missing in maize endosperm of the maize ae mutant, indicating a possible interaction of SBEI and SBEIIb. Seo et al. [24] found that when SBEs were heterologously expressed in a yeast system, SBEIIa and/or SBEIIb appear to act before SBEI on synthesizing glucan structure. The studies of Yao et al. [25,26] suggest that in the absence of SBEIIb, a reciprocal inhibition exists between SBEI and SBEIIa, and that the presence of either SBEI or SBEIIa increases amylopectin branching as opposed to the presence of SBEI and SBEIIa together.

Direct evidence for protein-protein interactions between SBEs and different members of all the proteins involved in starch biosynthesis has also been reported by several groups, based on co-immunoprecipitation and affinity purification methods. Tetlow et al. [27] reported that SBEI from wheat amyloplasts was present in a high molecular weight complex with starch phosphorylase and SBEIIb. A separate study [56] using maize amyloplasts showed that eliminating SBEIIb caused significant increases in the abundance of SBEI, BEIIa, SSIII, and starch phosphorylase in the granule, without affecting SSI or SSIIa. Hennen-Bierwagen [30] reported that SBEI and SSI were shown to interact in one of three independent methods tested; SBEI did not interact with any of the other proteins in their study (SSIIa, SSIII, SBEIIa, SBEIIb), and unlike the other five proteins in their study, SBEI was the only protein to exist as a monomer in gel permeation chromatography.

In present study, the sbe1a mutant line is nearly isogenic with the Wt control. Most if not all mutant phenotypes are likely the result of many effects, direct and indirect, on the overall growth, development and physiology of the plant, so it is impossible to truly isolate a primary effect of the mutation when looking at a whole plant level phenotype, even the starch structure phenotype. Modifying SBE activity may induce modifications in the distribution of phosphate groups within amylopectin such as in potato $[57,58]$. This may alter accessibility of amylase $(\alpha$ or $\beta$ ) to its substrate and may induce differences in digestibility. Nonetheless, there is value in observing and characterizing the phenotype of these plants, both at the macro and molecular levels as we have presented. We have a sister paper [36] which does investigate the effect of various SBE mutations on leaf starch which further sheds light on the SBEI function in the context of pleiotropic consequences.

\section{Evolution and Function of Maize SBEI Isoform in Starch Biosynthesis}

This work for the first time reports a specific and unique function for SBEI during the life cycle of maize. Molecular structure analysis suggests an important function of SBEI in modulating the branching pattern in normal starch by decreasing local clustering of amylopectin branch points. Thompson [59] emphasized the non-random nature of the distribution of branch points in starch. A specific type of non-random branching pattern may be required to optimize both storage and hydrolysis. It is reasonable to hypothesize that alteration in the specific non-random branching pattern could lead to an altered granule organization, rendering it more or 
less favorable to the plant for storage and/or for enzyme hydrolysis during utilization. Our data from in vitro starch digestibility and from plant germination analysis support this hypothesis.

Gene duplication and neo-functionalization are well known mechanisms by which specific genes can evolve to express different isoforms of enzymes with slightly specialized expression patterns or different enzymatic activities [60-62]. With the evidence from current and previous work, we can infer that an ancestral Sbe gene has duplicated at least twice during the evolution of maize, and these evolved to express three different SBE isoforms with highly specific functions in starch biosynthesis. A detailed phylogenetic analysis of the branching enzymes was published by Deschamps et al. [14]. This work demonstrated that genes belonging to both the SBEI and SBEII families can be identified in the green alga, which supports the theory that these two families of genes evolved approximately a billion years ago based on phylogenetic estimates of the divergence between the Chlorophyta and Magnolippyta lineages (estimates range from 729-1210 million years ago) $[63,64]$. This example of extreme evolutionary conservation is strong evidence for a specific and vital role for each enzyme isoform in starch biosynthesis. While most plant species studied retain genes representing each subfamily of SBE, Arabidopsis does not, suggesting that somewhere in the lineage leading to Arabidopsis, the gene was lost with minimal consequences to the species [65].

The evidence presented in this work strongly supports the hypothesis that SBEI is required to synthesize endosperm starch granules that allow normal hydrolysis and utilization during germination. Considering plant survival in the wild, optimal seedling vigor would be a strong evolutionary force to select for genotypes of plants with starch granules optimized for molecular structure that would lead to efficient storage and utilization. The reduced seedling vigor of sbe1a mutant seeds observed in this work provides powerful evidence for a specialized and important role of SBEI in plant development, consistent with the evolutionary conservation of SBEI in all higher plants.

\section{Conclusions}

This work for the first time reports that a lack of SBEI activity resulted in an observable effect, which was seen on both starch molecular structure and starch function. Structural and functional analysis of endosperm starch deficient in SBEI activity strongly supports the hypothesis that SBEI is required to synthesize starch granules for normal kernel development, allowing efficient hydrolysis and utilization.
Evidence from this work reveals a unique and essential function of SBEI in normal plant development, consistent with the evolutionary conservation of SBEI in all higher plants.

The new knowledge generated in this work will contribute to our understanding of the function and evolution of the maize SBEs, and of their roles in the biosynthesis, hydrolysis and utilization of starch granules. Moreover, the novel sbela starch might have application as a food ingredient with nutritional benefit.

\section{Methods}

\section{Starch Material}

Maize plants of Wt and sbe1a mutant were grown during summer, 2007 at Penn State Horticultural Research Farm (Rock Springs, PA). In order to compare starch material within a highly similar genetic background, homozygous Sbe1a/Sbe1a (i.e. Wt) and sbe1a/sbe1a mutant siblings were identified from a single segregating population derived from seeds of selfed Sbe1a/sbe1a plants to obtain ears for endosperm analysis. Genotyping of Wt and sbe1a mutant plants followed Blauth et al. [37]. The detected homozygous Wt and sbe1a mutant plants were self-pollinated to produce ears for endosperm analysis, and are segregated from a $\mathrm{BC}_{4} \mathrm{~F}_{3}$ population backcrossed by Blauth et al. [11,37]. Starch extraction from three different ears, considered as three biological replications, for each genotype, was according to Yao et al. [66]. Starch fractionation followed Klucinec and Thompson [67].

\section{$\beta$-Amylolysis of Amylopectin and Debranching of $\beta$ - Dextrins}

$\beta$-Dextrins were prepared by the method of Xia and Thompson [40] with slight modifications in sample size. Amylopectin samples (48 mg) were dispersed in $480 \mu \mathrm{L}$ of $90 \%$ dimethyl sulfoxide (DMSO) by heating in a boiling water bath for $10 \mathrm{~min}$. To the dispersion, warm sodium acetate buffer $\left(3.52 \mathrm{~mL}, 50^{\circ} \mathrm{C} 0.02 \mathrm{M}\right.$, $\mathrm{pH}$ 6.0) was added. The mixture was heated in a boiling water bath for $10 \mathrm{~min}$ and cooled to $50^{\circ} \mathrm{C}$. A 200$\mu \mathrm{L}$ aliquot of a $\beta$-amylase (from barley, Cat.No. EBARBL; Megazyme International Ireland, Ltd.) solution $(250 \mathrm{U} / \mathrm{mL}, 0.02 M$ sodium acetate, $\mathrm{pH} 6.0)$ was added, and the samples were incubated at $50^{\circ} \mathrm{C}$ with constant agitation (200 strokes/min). At approximately $10 \mathrm{~min}, 30 \mathrm{~min}, 1 \mathrm{~h}, 2 \mathrm{~h}, 6 \mathrm{~h}$, and $24 \mathrm{~h}$, a $0.5-\mathrm{mL}$ aliquot of sample was removed and heated in a boiling water bath for $10 \mathrm{~min}$ to stop the reaction. The procedures for precipitating $\beta$-dextrins and debranching $\beta$ dextrins by successive action of isoamylase (from Pseudomonas sp., Cat.No. E-ISAMY; Megazyme) and pullulanase (from Klebsiella planticola, Cat.No. E- 
PULKP; Megazyme) were the same as used previously for $\beta$-LDs) $[39,40]$.

\section{Preparation of Isoamylase-Debranched and Isoamylase plus Pullulanase-Debranched $\beta$-Limit Dextrins from Amylose Fractions}

The preparation and debranching of $\beta$-LDs followed the procedures in Klucinec and Thompson [39] with slight modifications in sample size. After the $\beta$-LDs were debranched with isoamylase for $24 \mathrm{~h}$, a $30-\mu \mathrm{L}$ aliquot of the digested solution was added to $270 \mu \mathrm{L}$ of DMSO and reserved for analysis by high-performance size-exclusion chromatography (HPSEC). Then the $\beta$-LDs were further debranched with pullulanase for $24 \mathrm{~h}$, afterwards another $30-\mu \mathrm{L}$ aliquot of the digested solution was added to $270 \mu \mathrm{L}$ of DMSO for HPSEC analysis [40].

\section{Resistant Starch Determination}

The official method for in vitro RS determination (AOAC 2002.02, AACC 32-40) was employed, which was scaled-down and modified for direct analysis of the digestion supernatant for total carbohydrate [41]. The modification allowed analysis of digestion time-course for small starch samples $(\sim 20 \mathrm{mg})$. For RS determination, after the $16 \mathrm{~h}$ digestion step at $37^{\circ} \mathrm{C}$ with porcine pancreatic $\alpha$-amylase and amyloglucosidase (enzymes from RS Assay Kit, Cat.No. K-RSTAR, Megazyme), the sample tube was removed from the water bath and to an aliquot of each sample was added 1 volume of $95 \%$ $(\mathrm{v} / \mathrm{v})$ ethanol with $0.5 \%(\mathrm{w} / \mathrm{v})$ EDTA. After centrifugation $(1,500 \times g, 10 \mathrm{~min})$, the supernatant was analyzed in duplicate for total carbohydrate using the phenol sulfuric acid method [68]. The percent non-digested starch (\% NDS) was calculated from this data and was the basis for the calculation of the RS value. Starch isolated from Wt and sbe1a mutant endospermes from three separate plants (triplicate biological replications) were subjected to triplicate pancreatic $\alpha$-amylase digestion, for determining the RS values.

\section{Digestion Time-Course Analysis}

For determination of digestion time-course, the starch samples were digested as described above. An aliquot was removed at approximately $30 \mathrm{sec}, 3 \mathrm{~min}, 6 \mathrm{~min}, 15$ min, $30 \mathrm{~min}, 1 \mathrm{~h}, 2 \mathrm{~h}, 3 \mathrm{~h}, 4 \mathrm{~h}, 5 \mathrm{~h}, 7 \mathrm{~h}, 10 \mathrm{~h}, 13 \mathrm{~h}$, and twice at $16 \mathrm{~h}$, and added to 1 volume of ethanol/EDTA solution to ensure immediate deactivation of the enzymes. After centrifugation the supernatants were analyzed for total carbohydrate as described above.

Digestion time-course was analyzed following the method developed by Rees [42] to obtain kinetic data. A "Double, 5 parameter" regression model in SigmaPlot (Systat Software, Inc.) was selected to fit the data using the double exponential decay equation:

$$
y=y_{0}+S_{1} e^{-k_{1} x}+S_{2} e^{-k_{2} x}
$$

where $y$ is \% NDS, $\mathrm{x}$ is the time, $y_{0}$ is the $y$-value that the model asymptotically approaches, $S_{1}$ and $S_{2}$ are the concentrations of the two different substrate components, and $k_{1}$ and $k_{2}$ are the reaction rate constants for the decay of the two different components. The units for $y_{0}, S_{1}$, and $S_{2}$ were $\%$ of initial starch, and the units for the rate constants were $\min ^{-1}$. After running the regression program, the software gives three possible completion status messages depending on how well the model fits the data:

(1) Converged, tolerance satisfied.

(2) Converged, tolerance satisfied. Parameter may not be valid. Array numerically singular on final iteration.

(3) Didn't converge, exceeded maximum number of iterations.

The data were kept for further regression analysis if message 1 or 2 resulted, and were discarded if message 3 resulted.

Digestion time-course analysis was performed for three biological replications per genotype. For each biological replication, two technical replications were performed. If both sets of data "converged" using the model (message 1 or 2), no further analyses were performed. If message 3 appeared, a new technical replication was done until the data "converged." The data from the two "converged" technical replications for each biological replication were combined, and the software program was run on the combined data. For all samples, the regression model fit for the combined data completed with convergence (Message 1), and generated valid parameters for analysis. Using the combined data, values for five parameters in the equation were determined for each biological replication. A mean and standard deviation of the five parameters for each genotype was then calculated, and comparisons among genotypes were made by one-way ANOVA analysis.

\section{Light Microscopy}

Bright field and polarized light microscopy were performed using a light microscope (BX51; Olympus) with an attached digital camera (Spot II RT; Diagnostic Instruments). $5 \mathrm{mg}$ of native starch sample was mixed with $0.5 \mathrm{~mL}$ of deionized water in a micro-centrifuge tube. For the resistant starch samples, the supernatant was removed after centrifugation of digestion solution and $20 \mu \mathrm{L}$ of deionized water was added to the pellets to disperse the sample. To examine the sample under the microscope, $20 \mu \mathrm{L}$ of the dispersed sample was added to a glass slide, and a cover slip was fixed over 
the sample with fingernail polish. Examination of iodinestained starch followed the method in Evans and Thompson [54]. $20 \mu \mathrm{L}$ of iodine solution $\left(0.08 \% \mathrm{I}_{2}\right.$, $0.12 \% \mathrm{KI}$ ) was placed onto $20 \mu \mathrm{L}$ of the dispersed sample to give a final $\mathrm{I}_{2}$ concentration of $0.04 \%$. In order to compare birefringence between granules, the camera's automatic exposure function was turned off, and the exposure was set the same for all samples. The same sample field was examined under bright field and polarized light.

Heterogeneity of iodine staining was evaluated quantitatively by a volunteer panel. Differentially iodinestained starch granules were classified into two categories, dark or light stained granules, and were sorted visually by five individual evaluators who were not otherwise involved in the research. The evaluators were trained to understand the difference between dark and light stained granules, by observing granules in a portion of a micrograph for sbe1a granules. Four micrographs for each genotype (Wt or sbe1a) were used for sorting. The evaluators were then given those eight micrographs, unlabeled and in randomized order, and asked to sort the granules into two categories. The proportion of dark granules for each micrograph was calculated based on the sorting results from all five evaluators, and a mean proportion was obtained for each micrograph. For each genotype, a mean was calculated from the means of the four micrographs. Comparison between two genotypes was made by one-way ANOVA.

\section{Scanning Electron Microscopy}

A thin layer of starch sample was applied to doublesided sticky carbon tape on a specimen stub, and sputter-coated with $10 \mathrm{~nm} \mathrm{Au/Pd} \mathrm{(BAL-TEC} \mathrm{SCD} \mathrm{050;} \mathrm{US-}$ TechnoTrade). Samples were then examined using a scanning electron microscope (JSM-5400; JEOL Ltd.) at an accelerating voltage of $20 \mathrm{keV}$ and at different magnification levels $(1,500 \times, 3,500 \times$, and $10,000 \times)$. For image collection, lower magnification was first employed to examine the whole view of samples, and higher magnification was then used to focus on sample areas that were representative overall.

\section{Kernel Germination Assay}

A kernel germination assay was performed according to the method in Dinges et al. [23] with slight modifications. Mature, dried maize kernels were surface-sterilized by immersion in $15 \mathrm{~mL}$ of $1 \%$ sodium hypochlorite for $5 \mathrm{~min}$ and then washed three times with deionized water. 15 kernels from each of three ears for each genotype were placed in Petri dishes containing three layers of moist Whatman paper and incubated at $30^{\circ} \mathrm{C}$ in the dark. The length of each coleoptile was measured by a ruler on successive days throughout the 11-day incubation period. To measure the amount of endosperm starch remaining, the roots, coleoptiles, embryo, and pericarps were removed from 2 kernels at days 1, 6, 8 , and 11 . The remaining endosperm was ground with a mortar and pestle on ice. The powered tissue was washed into a tube with deionized water and homogenized with a Tissumizer (Model SDT 1810; Tekmar) at $20,000 \mathrm{rpm}$ for $1 \mathrm{~min}$. The ground tissue was washed with deionized water, centrifuged at $1500 \times g$ for 10 $\mathrm{min}$, and suspended in $3 \mathrm{~mL}$ of deionized water. For calculating the dry weight of samples, $1 \mathrm{~mL}$ of this suspension was dried at $70^{\circ} \mathrm{C}$ overnight and weighed. The remaining $2 \mathrm{~mL}$ of the suspension was boiled for 30 min, and the total glucan polysaccharide in the solubilized solution was quantified in triplicates, using a commercial assay kit that measures glucose released after digestion with $\alpha$-amylase and amyloglucosidase (Cat.No. K-TSTA; Megazyme). The quantified starch content was normalized against the dry weight for comparison between genotypes.

\section{Additional material}

\section{Additional file 1: Chromatograms of isoamylase-debranched amylopectin and amylose fractions from Wt (- $)$ and sbe1a mutant (- - ) starch.}

Additional file 2: Difference plots between sbe1a mutant and $\mathrm{Wt}$ starch for the proportions of chains from debranched $\beta$-dextrins during time course of $\beta$-amylolysis of amylopectin. Individual plots for sbela mutant and Wt are presented in Figure $1 \mathrm{~A}$.

Additional file 3: Chain length distribution of isoamylasedebranched and isoamylase-plus-pullulanase-debranched $\beta$-limit dextrins from the amylopectin fraction from $\mathrm{Wt}$ and sbe1a mutant starch

Additional file 4: Chain length distribution of isoamylasedebranched and isoamylase-plus-pullulanase-debranched $\beta$-limit dextrins from the amylose fraction from $\mathrm{Wt}$ and sbe1a mutant starch.

Additional file 5: Bright field (left) and polarized light (right) micrographs of residual starch after $16 \mathrm{~h} \alpha$-amylase digestion from Wt and sbe1a mutant. Arrows point to residual granules with dark center.

Additional file 6: Transmission electron micrographs of residual starch after $16 \mathrm{~h} \alpha$-amylase digestion from Wt (left) and sbe1a mutant (right). Scale bars represent $5 \mu \mathrm{m}$ at the top of the graphs.

Additional file 7: Chromatograms of isoamylase-debranched resistant starch from Wt (- $)$ and sbe1a mutant (- - -) starch

\section{Acknowledgements}

We thank Yuan Yao and Jihong Li for their contributions to the breeding of maize sbe genotype into W64A background; we thank Missy Hazen at Electron Microscopy Facility at PSU for assistance with microscopy preparations; we thank fellow graduate students in Department of Food Science at PSU for their help in evaluating differentially-stained starch granules.

\section{Author details}

${ }^{1}$ MARS Petcare US, 315 Cool Springs Boulevard, Franklin, Tennessee 37067, USA. ${ }^{2}$ Department of Biochemistry, Biophysics \& Molecular Biology, lowa 
State University, Ames, lowa 50011-3260, USA. ${ }^{3}$ Department of Food Science, The Pennsylvania State University, University Park, Pennsylvania 16802-2504, USA. ${ }^{4}$ Department of Horticulture, The Pennsylvania State University, University Park, Pennsylvania 16802-5807, USA.

\section{Authors' contributions}

HX conceived of the study, performed the experiments, and drafted the manuscript. MYN participated in maize genotype breeding and discussion of experimental design and major results. DBT \& MJG advised the conception of the study, experimental design, result discussion, and revised the manuscript. All authors read and approved the final manuscript.

Received: 10 December 2010 Accepted: 21 May 2011

Published: 21 May 2011

\section{References}

1. Buléon A, Ball S, Planchot V, Colonna P: Starch granules: structure and biosynthesis. Int J Biol Macromol 1998, 23:85-112.

2. Gidley MJ: Starch structure/function relationships: achievements and challenges. In Starch: advances in structure and function. Edited by: Tina, et al. Royal Society of Chemistry (Great Britain). Food Chemistry Group; 2001:1-7.

3. Manners DJ: Recent developments in our understanding of amylopectin structure. Carbohydr Polym 1989, 11:87-112.

4. Boyer CD, Preiss J: Multiple forms of (1,4)-a-D-glucan-6-glucosyl transferase from developing Zea Mays L. kernels. Carbohydr Res 1978, 61:321-34.

5. Boyer $C D$, Preiss J: Evidence for independent genetic control of the multiple forms of maize endosperm branching enzymes and starch synthases. Plant Physiol 1981, 67:1141-5.

6. Gao M, Fisher DK, Kim KN, Shannon JC, Guiltinan MJ: Independent genetic control of maize starch-branching enzymes lla and Illb-Isolation and characterization of a Sbe2a cDNA. Plant Physiol 1997, 114:69-78.

7. Kim KN, Fisher DK, Gao M, Guiltinan MJ: Genomic organization and promoter activity of the maize Starch branching enzyme I gene. Gene 1998, 216:233-43.

8. Kim KN, Gao M, Fisher DK, Guiltinan MJ: Molecular cloning and characterization of the Amylose-Extender gene encoding starch branching enzyme IIB in maize. Plant Mol Biol 1999, 38:945-56.

9. Burton RA, Bewley JD, Smith AM, Bhattacharyya MK, Tatge H, Ring S, Bull V, Hamilton WDO, Martin C: Starch branching enzymes belonging to distinct enzyme families are differentially expressed during pea embryo development. Plant J 1995, 7:3-15.

10. Gao M, Fisher DK, Kim KN, Shannon JC, Guiltinan MJ: Evolutionary conservation and expression patterns of maize starch branching enzyme I and IIb genes suggests isoform specialization. Plant Mol Biol 1996, 30:1223-32.

11. Blauth SL, Yao Y, Klucinec JD, Shannon JC, Thompson DB, Guiltinan MJ: Identification of Mutator insertional mutants of starch-branching enzyme 2a in corn. Plant Physiol 2001, 125:1396-405.

12. Rahman S, Regina A, Li Z, Mukai Y, Yamamoto M, Kosar-Hashemi B, Abrahams S, Morell MK: Comparison of starch-branching enzyme genes reveals evolutionary relationships among isoforms. Characterization of a gene for starch-branching enzyme lla from wheat $D$ genome donor Aegilops tauschii. Plant Physiol 2001, 125:1314-24.

13. $\mathrm{Xu} \mathrm{JH}$, Messing J: Organization of the prolamin gene family provides insight into the evolution of the maize genome and gene duplication in grass species. Proc Natl Acad Sci 2008, 105:14330-35

14. Deschamps P, Moreau H, Worden AZ, Dauvillée D, Ball SG: Early Gene Duplication Within Chloroplastida and Its Correspondence With Relocation of Starch Metabolism to Chloroplasts. Genetics 2008, 178:2373-87.

15. Guan HP, Preiss J: Differentiation of the properties of the branching isozymes from maize (Zea mays). Plant Physiol 1993, 102:1269-73.

16. Takeda Y, Guan HP, Preiss J: Branching of amylose by the branching isoenzymes of maize endosperm. Carbohydr Res 1993, 240:253-63.

17. Boyer $C D$, Preiss J: Properties of citrate-stimulated starch synthesis catalyzed by starch synthase I of developing maize kernels. Plant Physiol 1979, 64:1039-42.

18. Gao M, Wanat J, Stinard PS, James MG, Myers AM: Characterization of dull 1, a maize gene coding for a novel starch synthase. Plant Cell 1998, 10:339-412.
19. Beatty MK, Rahman A, Cao H, Woodman W, Lee M, Myers AM, James MG: Purification and molecular genetic characterization of ZPU1, a pullulanase-type starch-debranching enzyme from maize. Plant Physiol 1999, 119:255-66.

20. Nishi A, Nakamura Y, Tanaka N, Satoh H: Biochemical and genetic effects of amylose-extender mutation in rice endosperm. Plant Physiol 2001, 127:459-72.

21. Colleoni C, Myers AM, James MG: One- and two-dimensional native PAGE activity gel analyses of maize endosperm proteins reveal functional interactions between specific starch metabolizing enzymes. J Appl Glycosci 2003, 50:207-12.

22. Dinges JR, Colleoni C, Myers AM, James MG: Molecular structure of three mutations at the maize sugary 1 locus and their allele-specific phenotypic effects. Plant Physiol 2001, 125:1406-18.

23. Dinges JR, Colleoni C, James MG, Myers AM: Mutational analysis of the pullulanase-type debranching enzyme of maize indicates multiple functions in starch metabolism. Plant Cell 2003, 15:666-80

24. Seo BS, Kim S, Scott MP, Singletary GW, Wong KS, James MG, Myers AM: Functional interactions between heterologously expressed starchbranching enzymes of maize and the glycogen synthases of Brewer's yeast. Plant Physiol 2002, 128:1189-99.

25. Yao Y, Thompson DB, Guiltinan MJ: Starch biosynthesis in maize endosperm: in the absence of SBEllb, the deficiency of SBElla leads to increased amylopectin branching. Presentation at 2003 AACC conference, Portland, $O R$.

26. Yao Y, Thompson DB, Guiltinan MJ: Maize starch branching enzyme (SBE) isoforms and amylopectin structure: in the absence of SBEllb, the future absence of SBEla leads to increased branching. Plant Physiol 2004, 106:293-316.

27. Tetlow IJ, Wait R, Lu Z, Akkasaeng R, Bowsher CG, Esposito S, KosarHashemi B, Morell MK, Emes MJ: Protein phosphorylation in amyloplasts regulates starch branching enzyme activity and protein-protein interactions. Plant Cell 2004, 16:694-708

28. Tetlow IJ, Beisel KG, Cameron S, Makhmoudova A, Liu F, Bresolin NS, Wait R, Morell MK, Emes MJ: Analysis of protein complexes in wheat amyloplasts reveals functional interactions among starch biosynthetic enzymes. Plant Physiol 2008, 146:1878-91

29. Deschamps $P$, Colleoni C, Nakamura Y, Suzuki E, Putaux JL, Buleon A, Haebel S, Ritte G, Steup M, Falcon L, Moreira D, Loffelhardt W, Nirmal RJ, Plancke C, d'Hulst C, Dauvillee D, Ball S: Metabolic symbiosis and the birth of the plant kingdom. Mol Biol Evol 2008, 25:536-48.

30. Hennen-Bierwagen TA, Liu F, Marsh R, Kim S, Gan Q, Tetlow IJ, Emes MJ, James MG, Myers AM: Starch biosynthetic enzymes from developing Zea mays endosperm associate in multisubunit complexes. Plant Physiol 2008, 146:1892-908.

31. Hennen-Bierwagen TA, Lin Q, Grimaud F, Planchot V, Keeling PL, James MG, Myers AM: Proteins from multiple metabolic pathways associate with starch biosynthetic enzymes in high molecular weight complexes: a model for regulation of carbon allocation in maize amyloplasts. Plant Physiol 2009, 149:1541-59.

32. Kötting O, Kossmann J, Zeeman SC, Lloyd JR: Regulation of starch metabolism: the age of enlightenment? Current Opinion in Plant Biology 2010, 13:321-9.

33. Liu F, Makhmoudova A, Lee EA, Wait R, Emes MJ, Tetlow IJ: The amylose extender mutant of maize conditions novel protein-protein interactions between starch biosynthetic enzymes in amyloplasts. J Exp Bot 2009, 60:4423-40.

34. Garwood DL, Shannon JC, Creech RG: Starches of endosperms possessing different alleles at the amylose-extender locus in Zea mays L. Cereal Chem 1976, 53:355-64.

35. Boyer CD, Daniels RR, Shannon JC: Starch granule (amyloplast) development in endosperm of several Zea mays L. genotypes affecting kernel polysaccharides. Amer J Bot 1977, 64:50-56

36. Yandeau-Nelson MD, Laurens L, Shi Z, Xia H, Smith AM, Guiltinan MJ: Starch Branching Enzyme lla is required for proper diurnal cycling of starch in leaves of Zea mays. Plant Physiology Online Open Access 2011 [http://www.plantphysiol.org/content/early/2011/04/20/pp.111.174094.full. pdf $+h t m l]$.

37. Blauth SL, Kim K, Klucinec JD, Shannon JC, Thompson DB, Guiltinan MJ: Identification of Mutator insertional mutants of starch branching enzyme 1 (sbe1) in Zea mays L. Plan Mol Bio 2002, 48:287-97. 
38. Xia H: Structure and function of endosperm starch from maize mutants deficient in one or more starch-branching enzyme isoform activities. Ph. D. thesis The Pennsylvania State University, University Park, PA; 2009.

39. Klucinec JD, Thompson DB: Note: Structure of amylopectins from aecontaining maize starches. Cereal Chem 2002, 79:19-23.

40. Xia H, Thompson DB: Debranching of $\beta$-limit dextrins with isoamylase or pullulanse to explore the Branching Pattern of Amylopectins from Three Maize Genotypes. Cereal Chem 2006, 83:668-76.

41. Evans A, Thompson DB: Enzyme susceptibility of high-amylose starch precipitated from sodium hydroxide dispersions. Cereal Chem 2008, 85:480-7.

42. Rees E: Effect of a heat-moisture treatment on alpha-amylase susceptibility of high amylose maize starches. MS thesis The Pennsylvania State University, University Park, PA; 2008.

43. Hizukuri S: Polymodal distribution of the chain lengths of amylopectins, and its significance. Carbohydr Res 1986, 147:342-7.

44. Lee EYC: The action of sweet potato $\beta$-amylase on glycogen and amylopectin: formation of a novel limit dextrin. Arch Biochem Biophys 1971, 146:488-92.

45. Takeda Y, Hizukuri S, Takeda C, Suzuki A: Structures of branched molecules of amyloses of various origins, and molar fractions of branched and unbranched molecules. Carbohydr Res 1987, 165:139-45.

46. Ao Z, Simsek S, Zhang G, Venkatachalam M, Reuhs BL, Hamaker BR: Starch with slow digestion property produced by altering its chain-length, branch density and crystalline structure. J Agri Food Chem 2007, 55:4540-7.

47. Li JH, Thompson DB, Guiltinan M: Mutation of the maize sbe1a and ae genes alters morphology and physical behavior of $w x$-type endosperm starch granules. Carbohydr Res 2007, 342:2619-27.

48. Zhang G, Ao Z, Hamaker BR: Slow digestion property of native cereal starches. Biomacromolecules 2006, 7:3252-8.

49. Leach HW, Schoch TJ: Structure of the starch granule. II. Action of various amylase on granular starches. Cereal Chem 1961, 38:34-46.

50. Valetudie JC, Colonna P, Bouchet B, Gallant DJ: Hydrolysis of tropical tuber starches by bacterial and pancreatic a-amylases. Starch/Stärke 1993, 45:270-6.

51. Helbert W, Schulein M, Henrissat B: Electron microscopic investigation of the diffusion of bacillus licheniformis a-amylase into corn starch granules. Int J Biol Macromol 1996, 19:165-9.

52. Gallant DJ, Bouchet B, Buleon A, Perez S: Physical characteristics of starch granules and susceptibility to enzymatic degradation. Eur J Clin Nutr 1992, 46:S3-S16.

53. Planchot V, Colonna P, Gallant DJ, Bouchet B: Extensive degradation of native starch granules by alpha-amylase from Apergillus fumigatus. $J$ Cereal Sci 1995, 21:163-71.

54. Evans A, Thompson DB: Resistance to a-Amylase digestion in four native high-amylose maize starches. Cereal Chem 2004, 81:31-7.

55. Smith AM, Zeeman SC, Smith SM: Starch Degradation. Annu Rev Plant Biol 2005, 56:73-98.

56. Grimaud F, Rogniaux H, James MG, Myers AM, Planchot V: Proteome and phosphoproteome analysis of starch granule-associated proteins from normal maize and mutants affected in starch biosynthesis. J Exp Bot 2008, 59:3395-406.

57. Blennow A, Hansen M, Schulz A, Jørgensen K, Donald AM, Sanderson J: The molecular deposition of transgenically modified starch in the starch granule as imaged by functional microscopy. J Struct Biol 2003, 143:229-41.

58. Blennow A, Wischmann B, Houborg K, Ahmt T, Jørgensen K, Engelsen SB, Bandsholm O, Poulsen P: Structure function relationships of transgenic starches with engineered phosphate substitution and starch branching. Int J Biol Macromol 2005, 36:159-68.

59. Thompson DB: On the non-random nature of amylopectin branching. Carbohydr Polym 2000, 40:223-39.

60. Gingerich DJ, Hanada K, Shiu SH, Vierstra RD: Large-scale, lineage-specific expansion of a bric-a-brac/tramtrack/broad complex ubiquitin-ligase gene family in rice. Plant Cell 2007, 19:2329-48.

61. Prokhnevsky Al, Peremyslov W, Dolja W: Overlapping functions of the four class XI myosins in Arabidopsis growth, root hair elongation, and organelle motility. Proc Natl Acad Sci 2008, 105:19744-9.

62. Saleh A, Alvarez-Venegasm R, Yilmaz M, Le O, Hou G, Sadder M, AlAbdallat A, Xia Y, Lu G, Ladunga I, Avramova Z: The Highly Similar
Arabidopsis homologs of trithorax ATX1 and ATX2 encode proteins with divergent biochemical functions. Plant Cell 2008, 20:568-79.

63. Hedges SB, Dudley J, Kumar S: TimeTree: A public knowledge-base of divergence times among organisms. Bioinformatics 2006, 22:2971-2.

64. Chaw SM, Chang CC, Chen HL, Li WH: Dating the monocot-dicot divergence and the origin of core eudicots using whole chloroplast genomes. J Mol Evol 2004, 58:424-41.

65. Dumeza S, Wattebleda F, Dauvilleea D, Delvallea D, Planchotb V, Ball SG, D'Hulsta C: Mutants of Arabidopsis Lacking Starch Branching Enzyme II Substitute Plastidial Starch Synthesis by Cytoplasmic Maltose Accumulation. Plant Cell 2006, 18:2694-709.

66. Yao Y, Guiltinan MJ, Shannon JC, Thompson DB: Single kernel sampling method for maize starch analysis while maintaining kernel vitality. Cereal Chem 2002, 79:757-762.

67. Klucinec JD, Thompson DB: Fractionation of high amylose maize starches by differential alcohol precipitation and chromatograph of the fractions. Cereal Chem 1998, 75:887-96.

68. Dubois M, Gilles KA, Hamilton JK, Rebers PA, Smith F: Colorimetric method for determination of sugars and related substances. Anal Chem 1956, 28:350-6.

doi:10.1186/1471-2229-11-95

Cite this article as: Xia et al:: Deficiency of maize starch-branching enzyme i results in altered starch fine structure, decreased digestibility and reduced coleoptile growth during germination. BMC Plant Biology 2011 11:95.

\section{Submit your next manuscript to BioMed Central and take full advantage of:}

- Convenient online submission

- Thorough peer review

- No space constraints or color figure charges

- Immediate publication on acceptance

- Inclusion in PubMed, CAS, Scopus and Google Scholar

- Research which is freely available for redistribution

Submit your manuscript at www.biomedcentral.com/submit
C Biomed Central 